\title{
Crianças e adolescentes com HIV/Aids: revisão de estudos sobre revelação do diagnóstico, adesão e estigma ${ }^{1}$
}

\author{
Camila Peixoto Pessôa Guerra ${ }^{2}$ \\ Eliane Maria Fleury Seidl \\ Universidade de Brasília, Brasilia-DF, Brasil
}

\begin{abstract}
Resumo: O artigo discute os temas revelação do diagnóstico, adesão e estigma relativos a crianças e adolescentes infectados pelo Vírus da Imunodeficiência Humana (HIV). A revisão da literatura incluiu artigos nacionais e internacionais, publicados a partir do ano 2000, e foi realizada por meio de buscas eletrônicas em bases de dados. Foram selecionados vinte e um artigos, duas dissertações de mestrado e cinco publicações institucionais. Concluiu-se que a revelação do diagnóstico tende a ser postergada pelos cuidadores. O medo do estigma pode dificultar a comunicação clara sobre a enfermidade e o tratamento, com repercussões negativas sobre os níveis de adesão de crianças e adolescentes soropositivos. Diante dos desafios identificados, pesquisas e recomendações são sugeridas no sentido de nortear a prática profissional de equipes de saúde que prestam assistência a essa clientela.
\end{abstract}

Palavras-chave: Síndrome da imunodeficiência adquirida, crianças, adolescentes, estigma.

\section{Children and adolescents with HIV/Aids: a review on disclosure of diagnosis, adherence and stigma}

\begin{abstract}
This study aims to present and discuss the themes disclosure of diagnosis, adherence and stigma related to children and adolescents infected with Human Immunodeficiency Virus (HIV). A bibliographical review of literature included Brazilian and international articles published since 2000 available in electronic databases. Twenty-one articles, two dissertations and five institutional publications were selected. We concluded that caregivers tend to postpone the disclosure of diagnosis. Fear of stigma may hinder open communication about the disease and treatment, with negative repercussions on adherence of children and adolescents with HIV. In the face of challenges identified in the study, some recommendations and research are suggested so to guide the practice of health professionals who deliver care to this clientele.
\end{abstract}

Keywords: Acquired immune deficiency syndrome, children, adolescents, stigma.

\section{Niños y adolescentes con VIH/Sida: un repaso sobre revelación del diagnóstico, adhesión y estigma}

\begin{abstract}
Resumen: El artículo presenta y discute los temas revelación del diagnóstico, adhesión y estigma relacionados a niños y adolescentes infectados por el Virus de la Inmunodeficiencia Humana (VIH). El repaso bibliográfico de artículos nacionales e internacionales, publicados desde el año 2000, se realizó mediante búsquedas electrónicas en bases de datos. Fueron seleccionados veintiuno artículos, dos disertaciones y cinco publicaciones institucionales. Llegamos a la conclusión de que la revelación del diagnóstico suele ser aplazada por los familiares por una diversidad de factores. El temor del estigma puede dificultar la comunicación correcta sobre la enfermedad y el tratamiento, trayendo consecuencias negativas en la adhesión de niños e adolescentes con VIH. Delante de los desafíos citados, averiguaciones y recomendaciones son propuestas para conducir la práctica de los profesionales de equipos de salud que trabajan con esta población.
\end{abstract}

Palabras clave: Sindrome de inmunodeficiencia adquirida, niños, adolescentes, estigma.

O advento de medicamentos anti-retrovirais tem acarretado melhora relevante nas condições de saúde de crianças e adolescentes infectados pelo Vírus da Imunodeficiência Humana (HIV), permitindo ganhos no processo de desenvolvimento e na qualidade de vida. Ao contrário do que ocorria no início da epidemia da Síndrome da Imunodeficiência Adquirida (Aids), atualmente um grande número de crianças infectadas por transmissão vertical - transmissão do HIV da mãe para o bebê durante a gestação, parto ou aleitamento - atinge a adolescência e a idade adulta (Brown \& Lourie, 2000; Thorne e cols., 2002).

1 Apoio: CAPES. Este artigo é parte da dissertação de mestrado realizada pela primeira autora sob orientação da segunda.

2 Endereço para correspondência:

Camila Peixoto Pessôa Guerra. SQN 214, Bloco C, apto. 311. CEP

70873-030. Brasília-DF, Brasil.E-mail: cppguerra@gmail
Ainda que crianças e adolescentes vivendo com HIV/ Aids possam apresentar retardo puberal, alterações no desenvolvimento neuro-cognitivo e sequelas de doenças oportunistas anteriores, é crescente o número dos que apresentam crescimento físico e desenvolvimento emocional e cognitivo esperados para a idade (Brasil, 2007). Para tanto, é fundamental que crianças soropositivas para o HIV tenham acesso a acompanhamento médico regular, essencial para a prescrição do tratamento anti-retroviral de modo adequado e oportuno. Para os adolescentes, a prescrição dos anti-retrovirais deve ser adaptada ao estadiamento da puberdade: os que estão nas fases iniciais devem ser tratados de acordo com as recomendações pediátricas, enquanto que os jovens com maturação sexual mais adiantada devem ser tratados segundo as recomendações estabelecidas para adultos. Nas fases intermediárias, o tratamento deve ser individualizado 
a critério médico (Pinto, 2004). As características peculiares desse grupo etário impõem desafios para as equipes de saúde que prestam assistência a adolescentes soropositivos.

Ao lado da relevância das questões biomédicas, ainda se constata a presença de desafios na esfera psicossocial, tais como a revelação do diagnóstico, a adesão ao tratamento e o medo do estigma (Ayres e cols., 2006; Brackis-Cott, Mellins, Abrams, Reval, \& Dolezal, 2003; Pequegnat, 2002). Esses temas têm sido recorrentes na prática clínica de profissionais de saúde que assistem crianças/adolescentes com HIV/Aids e seus cuidadores, motivando estudos na área (Seidl, Rossi, Viana, Meneses, \& Meireles, 2005).

O presente artigo objetiva apresentar e discutir questões psicossociais relativas a crianças e adolescentes infectados pelo HIV, em especial por transmissão vertical, com destaque para revelação do diagnóstico, adesão ao tratamento e estigma. $\mathrm{O}$ trabalho se baseou em levantamento bibliográfico de artigos nacionais e internacionais, além de dissertações/ teses brasileiras que abordavam os temas de interesse.

\section{Método}

A revisão da literatura foi feita nas bases de dados EBSCOhost, Ovid, Medscape, ScienceDirect, SAGE, SciELO e PsycInfo. Foram selecionados 21 artigos (dois nacionais e dezenove internacionais) e duas dissertações de mestrado, excluídas as publicações repetidas que surgiram em mais de uma base de dados. Os artigos foram selecionados a partir de sua pertinência aos temas tratados e foram considerados aqueles publicados a partir do ano 2000 , tendo em vista a maior atualidade dos trabalhos. Foram incluídas ainda cinco publicações institucionais que abordavam os temas de interesse. Os descritores utilizados na busca eletrônica foram, em língua portuguesa: crianças com HIV/Aids, adolescentes com HIV/Aids, adesão ao tratamento, revelação do diagnóstico e estigma. Em língua inglesa, os descritores foram: children living with HIV/Aids, youth living with HIV/Aids, treatment adherence, disclosure e stigma.

Com base nos principais resultados e conclusões dos estudos analisados, são apontadas diretrizes e recomendações que visam a nortear a prática profissional das equipes de saúde que atuam junto a esse segmento de pessoas vivendo com HIV/Aids.

\section{Revelação de diagnóstico}

Estudos indicam que familiares e cuidadores de crianças e adolescentes soropositivos para o HIV tendem a postergar o momento da revelação do diagnóstico (Abadia-Barrero \& LaRusso, 2006; Gerson e cols., 2001; Grecca, 2004; Instone, 2000; Mellins e cols., 2002; Seidl e cols., 2005). Os motivos que os cuidadores apresentam para adiar a revelação dizem respeito às crianças ou aos próprios cuidadores. Dentre as razões atribuídas às crianças estão a imaturidade cognitiva para compreender a doença, a falta de questionamento ou curiosidade, a possível reação psicológica negativa da criança e o risco de esta falar sobre a condição para terceiros e ser vítima de preconceito. Quanto aos motivos referentes aos cuidadores, destacam-se o desconforto diante de eventual exposição da história familiar (a soropositividade dos pais, por exemplo), o medo do estigma, sentimentos de culpa pela transmissão do HIV e o sentimento de despreparo para abordar o assunto com a criança ou o adolescente (Bachanas e cols., 2001; Gerson e cols., 2001; Instone, 2000; Marques e cols., 2006; Seidl e cols., 2005).

A demora excessiva na revelação do diagnóstico pode levar à descoberta por outras vias, aumentando as chances de as informações sobre a enfermidade serem incorretas e imprecisas, podendo ocasionar confusão e desconfiança (Mellins e cols., 2002). O silêncio prolongado e a pobreza na comunicação também podem resultar em dificuldades de adesão, distúrbios de comportamento, estigma auto-dirigido e maiores níveis de distress psicológico das crianças/adolescentes e dos cuidadores, sobrecarregando a família como um todo (Abadía-Barrero \& LaRusso, 2006; Bachanas e cols., 2001; Grecca, 2004). O segredo sobre o diagnóstico pode ter ainda outras repercussões negativas, como a adoção de comportamentos sexuais de risco pelos adolescentes (Pequegnat, 2002).

Mesmo quando há conhecimento acerca da sorologia, a comunicação sobre a condição de soropositividade entre cuidadores e crianças/adolescentes muitas vezes não é aberta e franca. Abadia-Barrero e LaRusso (2006) pesquisaram a revelação do diagnóstico para crianças e adolescentes soropositivos que viviam em uma casa de apoio em São Paulo-SP. A pesquisa foi realizada com 36 crianças e adolescentes mediante a utilização de entrevistas e de observação participante. Os autores verificaram que não havia comunicação clara sobre HIV/Aids na casa. Apesar de as crianças e os adolescentes tomarem os medicamentos, não se conversava sobre a condição de saúde que motivava o uso dos anti-retrovirais. Destaca-se que falar mais abertamente sobre a enfermidade possibilita, entre outros benefícios, mais acesso a fontes de apoio social, reduzindo os efeitos negativos da experiência de ter uma doença crônica (Wiener \& Battles, 2006).

Em outro estudo, dois terços dos 77 cuidadores entrevistados relataram que as crianças sob sua responsabilidade não tinham conhecimento sobre o diagnóstico (Mellins e cols., 2002). Segundo os participantes, $59 \%$ das crianças acreditavam que tinham outro tipo de doença. Além da dificuldade em revelar o diagnóstico às crianças e aos adolescentes, existe também o medo de compartilhar essa informação com outras pessoas. Nessa perspectiva, Wiener e Battles (2006) categorizaram três níveis de revelação sobre o diagnóstico: baixo, médio e alto. O nível baixo de revelação inclúa situações nas quais apenas a família nuclear e/ou amigos próximos sabiam do diagnóstico. $\mathrm{O}$ nível mediano dizia respeito à revelação a todos os familiares e amigos em geral. O nível mais alto de revelação ocorria quando a maior parte das pessoas tinha conhecimento do diagnóstico. As autoras concluíram que 
os adolescentes que vivenciavam níveis altos de revelação eram mais competentes nas relações interpessoais com seus pares.

Apesar da forte resistência dos cuidadores em revelar o diagnóstico, há algumas situações que parecem motivá-los a romper o silêncio. Marques e cols. (2006) identificaram três categorias principais de razões para que a comunicação do diagnóstico ocorra, segundo relatos dos cuidadores. A primeira diz respeito a fatos que indicam a necessidade urgente de realizar a revelação, por exemplo, dificuldades de adesão, proximidade do início da vida sexual ou quando a criança pergunta muito sobre seu estado de saúde. Outro motivo identificado foi a crença de que a revelação traria benefícios à criança/adolescente. Nesse caso, os cuidadores acreditavam que manter o diagnóstico em segredo limitava as oportunidades da criança e do adolescente de lidar melhor com a soropositividade. Alguns cuidadores relataram também que a revelação tinha ocorrido na tentativa de corrigir uma intervenção profissional considerada inadequada, objetivando reduzir os possíveis danos ocasionados por uma informação diagnóstica pouco cuidadosa (Marques e cols., 2006).

Manter uma comunicação aberta e franca sobre a doença pode resultar em melhor relação familiar, melhor ajustamento psicossocial e enfrentamento por parte das crianças e menos ansiedade para os cuidadores (Abadia-Barrero \& LaRusso, 2006). O sentimento de alívio dos cuidadores após a revelação do diagnóstico é frequentemente observado na prática clínica, o que vai ao encontro dos resultados de Grecca (2004). A autora entrevistou cuidadores que haviam revelado o diagnóstico $(n=5)$ e cuidadores que não haviam feito a revelação $(n=24)$. Dentre os que haviam comunicado o diagnóstico aos filhos, quatro relataram sentimentos positivos e que se sentiram aliviados, e um relatou sentimentos de culpa por ter revelado.

A necessidade de dar voz aos próprios pacientes infanto-juvenis sobre essas questões não tem sido reconhecida, na medida em que há predomínio de estudos com os cuidadores. Com esse propósito, Abadia-Barrero e LaRusso (2006) realizaram entrevistas com as crianças que conheciam seu diagnóstico. De acordo com os resultados, as crianças não relataram sentimentos de raiva, preocupação, estresse, tristeza ou confusão no momento da pesquisa. Os cuidadores, por outro lado, avaliaram que ainda persistiam nas crianças reações de raiva e estresse, porém identificavam mais esperança e menos tristeza. Esses dados são ilustrativos das vantagens potenciais da revelação.

Marques e cols. (2006) argumentam que a revelação é necessária e irremediável, que a manutenção do segredo é desconfortável e angustiante, e recomendam que a equipe de saúde identifique profissionais que possuam maior vinculação com os pacientes para utilizá-los como referência. Dessa forma, o profissional que tiver um melhor relacionamento com o paciente poderá auxiliar o processo da revelação à criança ou ao adolescente com HIV/Aids. O estudo desses autores mostrou que as demandas para a revelação vêm da família e/ ou da equipe de saúde. Foi frequente, entre os adolescentes entrevistados, a opinião de que a revelação deveria ocorrer o mais cedo possível, com o devido cuidado e de forma clara. Outro aspecto constatado pelos pesquisadores é que a comunicação do diagnóstico para os adolescentes que cresceram com HIV mostrou-se cercada de cuidados, ao passo que não houve a mesma preocupação com jovens que se infectaram pela via sexual.

Constata-se que a revelação do diagnóstico permite que crianças e jovens compreendam melhor a doença, exercendo papel ativo no tratamento. Além disso, se estes não sabem sobre seu diagnóstico, a possibilidade de que usufruam apoio social construtivo fica diminuída, na medida em que a não-revelação dificultaria o acesso a recursos psicossociais fundamentais para o ajustamento à condição de enfermidade crônica. Estudos mostram que a disponibilidade de apoio social tem impacto positivo na saúde mental e que intervenções da equipe de saúde junto a crianças e adolescentes infectados pelo HIV deveriam ter por objetivo auxiliar no fortalecimento do suporte social (Grecca, 2004).

Diretrizes para a revelação de diagnóstico sugerem que esta ocorra de forma processual (Gerson e cols., 2001). Inicialmente propõe-se que o profissional de saúde compartilhe informações com os cuidadores a respeito da família e da criança/adolescente, visando ainda à construção da confiança entre ambos. Em um segundo momento, é importante avaliar o nível de conhecimento dos cuidadores sobre HIV/ Aids e o tratamento, seguido de atividades educativas e de fornecimento de informações sobre esses aspectos para os cuidadores. Em seguida, o profissional de saúde deve avaliar, juntamente com os cuidadores, o momento e a maneira de proceder à revelação. Após um planejamento cuidadoso do momento considerado adequado, a meta é o evento propriamente dito: como, quando e quem participará da revelação. Por último, é muito importante o monitoramento do momento pós-revelação, que visa a identificar o impacto psicológico da revelação na família e no paciente, mediante a observação direta do comportamento da criança/adolescente e por relatos dos cuidadores. Assim, comunicar o diagnóstico de soropositividade deve ser um diálogo contínuo entre o paciente, a família e a equipe de saúde. Gerson e cols. (2001) assinalam ainda a necessidade de que haja tempo suficiente para o atendimento no qual ocorrerá a revelação do diagnóstico, de modo que a criança se sinta confortável para fazer perguntas e expressar sentimentos.

O profissional de saúde que atua junto a crianças/adolescentes soropositivos e suas famílias poderia se beneficiar de uma capacitação que o preparasse para a abordagem de temas como a revelação do diagnóstico. Tal capacitação deveria fornecer as ferramentas - técnicas, relacionais e de comunicação - necessárias para que o profissional de saúde procedesse a uma avaliação mais cuidadosa de cada caso. Por meio dessa avaliação, o profissional poderia identificar o nível de conhecimento e o preparo psicológico dos cuidadores para a revelação. É possível que um nível adequado de 
conhecimento sobre aspectos do HIV/Aids e do tratamento, além das vantagens da revelação, traga mais segurança aos cuidadores para abordar o assunto com suas crianças e adolescentes. Ademais, medos e inseguranças dos cuidadores podem ser dirimidos a partir de maior acesso a essas informações.

\section{Adesão à terapia anti-retroviral}

Estudos que abordam a adesão ao tratamento de crianças e jovens soropositivos para o HIV são escassos, além de haver informação insuficiente sobre as estratégias que podem melhorar a adesão desses pacientes (Byrne, Honig, Jurgrau, Heffernan, \& Donahue, 2002; Berrien, Salazar, Reynolds, \& McKay, 2004; Naar-King e cols., 2006). De acordo com a definição da World Health Organization (2003), a adesão é a extensão na qual o comportamento de uma pessoa coincide com o que foi acordado com a equipe de saúde (tomar remédios, fazer exercícios, seguir uma dieta). Dessa forma, adesão a tratamento implica negociação entre paciente e profissionais, e não um mero cumprimento de instruções.

A adesão ao tratamento em HIV/Aids guarda semelhanças com a conduta de adesão em outras doenças crônicas. Ademais, assim como algumas enfermidades crônicas, a Aids tem tratamento, mas não tem cura. No entanto, a adesão de pacientes pediátricos aos anti-retrovirais é complexa pela quantidade de medicamentos prescritos, pelo sabor às vezes considerado desagradável, além do fato de ser um tratamento para toda a vida. Outro desafio se refere à capacidade de o cuidador lembrar-se corretamente das medicações a serem dadas, suas quantidades e horários (Brackis-Cott e cols., 2003; Byrne e cols., 2002). Especificamente sobre a adesão de adolescentes à terapia anti-retroviral (TARV), esta pode sofrer a influência de peculiaridades observadas nessa faixa etária, como negação da enfermidade, desinformação, comprometimento da auto-estima, dificuldades de obter apoio social, entre outras (Brasil, 2007). Estudo de Seidl e cols. (2005) verificou a ocorrência de três categorias principais de dificuldades de adesão, segundo relatos de cuidadores de crianças e adolescentes soropositivos: (a) horários da medicação, em especial quando o medicamento tinha que ser tomado muito cedo ou quando a criança estava fora do ambiente doméstico (por exemplo, na escola); (b) gosto desagradável da medicação e/ou presença de efeitos colaterais, de modo que o tratamento constituía uma condição aversiva para a criança; (c) ocorrência de comportamentos oposicionistas da criança/adolescente como resistência à ingestão da medicação, muitas vezes por não compreender sua necessidade.

Dificuldades de adesão têm sido observadas mais frequentemente em adolescentes do que em crianças. Esse fato ocorre devido a atitudes de questionamento e de rebeldia manifestadas pelos adolescentes frente à soropositividade, ao tratamento e à supervisão dos cuidadores. Administrar a medicação às crianças é papel primordial dos cuidadores, enquanto que, no caso dos adolescentes, a responsabilidade no uso dos anti-retrovirais passa a ser apenas deles ou é compartilhada entre estes e seus familiares. Ocorre que, muitas vezes, os cuidadores transferem a responsabilidade da administração da medicação à criança ou ao adolescente apenas devido à idade (Martin e cols., 2007). Ao fazer essa transição baseando-se apenas na idade, em detrimento da responsabilidade para com a ingestão dos medicamentos, podem ocorrer problemas de adesão. Os autores sugerem que essa responsabilidade não dependa unicamente da idade do paciente, e sim do seu comprometimento com o próprio tratamento.

Dificuldades de adesão são comuns entre pessoas com doenças crônicas. Dessa forma, é importante identificar as variáveis associadas ao comportamento de adesão para possibilitar intervenções mais eficazes. Naar-King e cols. (2006) investigaram as associações entre adesão, auto-eficácia, suporte social e distress psicológico em jovens vivendo com HIV/Aids. Os resultados indicaram que, consistentemente com a literatura relativa a adultos, auto-eficácia e distress estiveram correlacionados à adesão medicamentosa. $\mathrm{O}$ primeiro teve associação positiva, isto é, quanto maior a percepção de auto-eficácia para realizar o tratamento, mais altos foram os níveis de adesão relatados. Já o distress apresentou correlação negativa com a adesão. Os autores sugerem que as ações médicas e psicossociais para melhorar a adesão com essa clientela devem aumentar o sentimento de auto-eficácia e reduzir o distress emocional.

A adesão não depende apenas do indivíduo portador da doença: no caso de crianças e adolescentes soropositivos para o HIV, esse comportamento também é de responsabilidade de seu cuidador. Nessa perspectiva, para atingir níveis satisfatórios de adesão, é essencial que haja uma boa adesão também do cuidador (Brackis-Cott e cols., 2003; Branco, 2007; Brasil, 2007). Assim, ações de saúde devem ser direcionadas aos familiares, em especial nos casos de crianças com faixa etária reduzida. Martin e cols. (2007) observaram que o maior conhecimento do cuidador acerca do esquema terapêutico esteve correlacionado a níveis adequados de adesão. Por conseguinte, uma maneira eficaz de intervir na adesão de crianças ou adolescentes soropositivos para o HIV é oferecer informações claras e organizadas aos cuidadores em relação ao esquema terapêutico.

Apesar das dificuldades para manter níveis satisfatórios de adesão, há diversas estratégias para tentar saná-las. Algumas das estratégias para melhorar a administração dos medicamentos e a adesão são: associar alimentos ao uso dos medicamentos; contar os comprimidos para monitorar a ingestão; utilizar diários, alarmes, calendários para evitar o esquecimento; revelar o diagnóstico e lançar mão de estratégias de convencimento sobre a necessidade de uso dos anti-retrovirais (Byrne e cols., 2002; Seidl e cols., 2005).

Há outras estratégias que podem ser utilizadas pelos profissionais de saúde, como as visitas domiciliares. Berrien e cols. (2004) conduziram um estudo com o objetivo de melhorar a adesão ao tratamento anti-retroviral de pacientes soropositivos de até 20 anos de idade. Os participantes foram 
divididos em grupo controle - que continuaria a receber os cuidados habitualmente oferecidos pelo serviço - e grupo de intervenção. A intervenção foi realizada nas casas dos pacientes e consistia de oito visitas estruturadas num período de três meses por um profissional de enfermagem com experiência em atendimentos domiciliares. As intervenções visavam a melhorar o nível de conhecimento sobre HIV/Aids e modificação ou extinção de barreiras à adesão. A adesão foi mensurada por auto-relato, registros da farmácia indicando se a medicação tinha sido retirada e pela contagem de linfócitos T CD4 e da carga viral plasmática. Os pesquisadores identificaram melhora significativa nos níveis de adesão, de conhecimento e no histórico de retirada de medicamentos nos adolescentes que foram submetidos à intervenção, concluindo que os atendimentos domiciliares foram uma estratégia eficaz para aumentar a adesão de adolescentes com HIV/ Aids.

O Ministério da Saúde (Brasil, 2007) destaca ainda as estratégias para promover a adesão e o acompanhamento clínico de adolescentes vivendo com HIV/Aids. Preparação do adolescente para aceitar melhor seu diagnóstico, negociação do tratamento com o compromisso do jovem, envolvimento e participação da família e de amigos no tratamento, criação de grupos de discussão e a escolha de um regime terapêutico que seja viável e mais compatível com seu estilo de vida são algumas das estratégias sugeridas.

\section{Estigma em HIV/Aids}

Estigma em HIV/Aids pode ser definido como a desvalorização de pessoas vivendo com essa condição (United Nations Programme on HIV/Aids [UNAIDS], 2007). A Aids é uma doença estigmatizada, e pessoas acometidas muitas vezes são vítimas de discriminação e preconceito (Pequegnat, 2002). Essa realidade pode ser confirmada na prática clínica em relatos de cuidadores e dos próprios adolescentes com HIV/Aids. Uma de suas consequências é o adiamento da revelação do diagnóstico devido ao medo da ocorrência do preconceito e discriminação. Desse modo, forma-se um círculo vicioso: o estigma tende a levar a mais sofrimento, que por sua vez resulta no silêncio sobre o assunto. E o segredo pode favorecer que o estigma ganhe espaço (Duffy, 2005).

O estigma é mais presente na Aids que em outras doenças crônicas, como o câncer ou a esclerose múltipla (Pequegnat, 2002). Segundo a autora, isso ocorre devido a quatro características da Aids que favorecem o estigma: (a) é uma doença percebida como letal; (b) é uma enfermidade que pode colocar outras pessoas em risco; (c) pode ser uma condição aparente a terceiros; e (d) é uma doença cujas causas são percebidas como de responsabilidade do indivíduo. Complementando essa caracterização, Gerson e cols. (2001) assinalam que o HIV/Aids elicia o estigma devido às formas comuns de transmissão do vírus: relações sexuais e uso de drogas injetáveis.
O estigma é um tópico recorrente nos discursos de jovens soropositivos e seus cuidadores. Ayres e cols. (2006) realizaram entrevistas com adolescentes soropositivos para o HIV e seus cuidadores. Pela análise desses relatos, os autores definiram duas categorias de estigma: sentido e decretado (enacted stigma). O primeiro diz respeito aos sentimentos subjetivos de vergonha associados à discriminação social e internalizados como parte da identidade. O estigma sentido esteve presente nos discursos dos cuidadores com relação aos medos das reações pós-revelação por parte dos adolescentes. Nos discursos dos jovens, esse tipo de sentimento foi evidenciado ao relatarem o medo de contar seus diagnósticos para amigos e parceiros. O estigma sentido ocorre quando o indivíduo tem receio ou mesmo antecipa a rejeição de terceiros e manifesta um sentimento de vergonha (Swendeman, Rotheram-Borus, Comulada, Weiss, \& Ramos, 2006). Segundo esses autores, essa modalidade de estigma pode levar as pessoas a moldarem seus comportamentos de modo a evitar o estigma decretado, que diz respeito às experiências reais de discriminação, sendo mais comum nos relatos verbais de jovens que contraíram a doença mediante relações sexuais ou uso de drogas injetáveis

O estigma traz uma série de consequências que podem ser tão devastadoras quanto a doença em si: abandono, ostracismo, perda de emprego, violência (Swendeman e cols., 2006; UNAIDS, 2007). E é justamente o medo do estigma que faz com que as pessoas não revelem sua condição. Porém, ao fazer isso, estas estariam limitando oportunidades para obtenção de apoio, de contatos sociais e até mesmo de tratamento.

Klunklin e Harrigan (2002) observaram consequências importantes do preconceito direcionado a crianças e adolescentes com HIV/Aids: muitas vezes estes têm dificuldades para se matricular em escolas regulares. Contudo, a ocorrência desse tipo de preconceito na escola não parece ser uma realidade generalizada. Seidl e cols. (2005) constataram que foram quase nulos os relatos de discriminação direcionados a crianças que tiveram seu status sorológico revelado na escola, segundo os cuidadores primários entrevistados. Os autores consideraram que provavelmente esteja ocorrendo uma mudança sócio-cultural importante, com tendência à diminuição desse tipo de atitude preconceituosa na realidade brasileira.

Para melhor lidar com a ocorrência do estigma, é necessário facilitar a discussão, a troca de vivências e desenvolver estratégias educativas com informações claras (Ferreira \& Gadelha, 2004; Marques e cols., 2006). Ayres e cols. (2006) também fazem sugestões sobre esse tema, apresentando uma série de recomendações para abordar as necessidades dos jovens soropositivos, como o aumento de informações e a expansão dos debates sobre estigma e discriminação relacionados à Aids.

O estigma é um dos principais motivos citados para que os cuidadores adiem a revelação do diagnóstico. Ressalta-se 
que é essencial que os cuidadores reconheçam seus direitos - inclusive o de manter essa informação em sigilo - para que possam se sentir mais seguros e amparados ao enfrentar situações dessa natureza. O profissional de saúde deve instrumentalizar os pacientes e seus cuidadores para que estejam a par desses direitos e os exerçam. Intervenções direcionadas à diminuição do estigma dependem de amplas modificações sócio-culturais. Dessa forma, destaca-se a relevância de iniciativas governamentais e não governamentais que esclareçam a população acerca dos mitos e verdades sobre o HIV/Aids, contribuindo para a diminuição do preconceito.

\section{Considerações finais}

Os temas apresentados e discutidos no presente artigo - revelação do diagnóstico, adesão ao tratamento e estigma são interligados: o medo do estigma associado ao HIV/Aids dificulta o processo de revelação, enquanto a comunicação sobre o diagnóstico é postergada muitas vezes devido ao medo do estigma. Dificuldades de adesão, por sua vez, podem ser consequência do desconhecimento do diagnóstico, enquanto este é consequência do receio dos cuidadores de que a criança ou o adolescente compartilhe essa informação com outras pessoas, quebrando o sigilo e possibilitando a ocorrência de estigma. Os resultados dos estudos analisados dão subsídios para práticas e intervenções médicas e psicossociais a serem implementadas por profissionais de saúde que prestam assistência a esse segmento de pessoas vivendo com HIV/Aids.

Pesquisas têm apontado as vantagens da revelação do diagnóstico para crianças e adolescentes com HIV/Aids, mas os cuidadores têm adiado essa decisão independente das recomendações das equipes de saúde. A não-revelação, por outro lado, parece trazer consequências negativas. A experiência de atendimento a essa clientela e os resultados de estudos sobre o tema indicam que não há associação entre conhecimento do diagnóstico e ocorrência de problemas de ajustamento psicossocial. Dessa forma, a revelação do diagnóstico, conduzida de forma adequada e planejada, pode favorecer a adaptação de crianças e adolescentes à soropositividade, propiciando que estes desenvolvam habilidades de enfrentamento para lidar com essa condição. Ademais, a partir do momento que têm informações corretas e realistas sobre a enfermidade, esses pacientes podem participar efetivamente do seu próprio tratamento e compreender a importância de aderir adequadamente à medicação.

A revelação do diagnóstico é, sem dúvida, um dos temas mais abordados nos estudos que investigaram crianças e adolescentes com HIV/Aids. Entretanto, é essencial pesquisar não apenas a ocorrência da revelação, mas sim a maneira como esta foi realizada e as consequências positivas e negativas advindas dessa comunicação. Já que a população de jovens soropositivos infectados por transmissão vertical apresenta sobrevida maior e com mais qualidade de vida, surge a oportunidade de realização de estudos longitudinais, que poderiam investigar temas como vivência da sexualidade, adoção de práticas sexuais seguras, enfrentamento do estigma e relacionamento entre pares, questões que têm emergido no atendimento a essa clientela.

$\mathrm{O}$ tema adesão merece investigações futuras, visto que esta deve atingir níveis bastante elevados para que o tratamento anti-retroviral seja eficaz. É importante compreender se o comportamento de adesão apresenta características típicas nas faixas etárias. Sugerem-se estudos com vistas a desenvolver programas e estratégias de intervenção mais específicas e que atendam melhor às demandas de adesão desse segmento de pessoas vivendo com HIV/Aids.

Ao contrário da revelação do diagnóstico, ou da adesão ao tratamento, que podem ser avaliadas e verificadas pelo profissional de saúde, o estigma está difundido em várias esferas da vida da pessoa soropositiva. Dessa forma, é mais difícil intervir sobre o estigma, se comparado a outros aspectos tratados no presente trabalho. Nessa perspectiva, é fundamental que haja ações governamentais e não governamentais e reforço das políticas públicas para que o estigma em relação ao HIV/Aids possa ser combatido na sociedade brasileira.

Além dos estudos teóricos, é de extrema relevância a condução de pesquisas que realizem ou proponham intervenções para promover a revelação do diagnóstico, o comportamento de adesão e o enfrentamento do estigma entre crianças e adolescentes soropositivos. Há também a necessidade de construção de instrumentos (escalas, inventários) específicos para essa população, uma vez que permitiria a avaliação mais adequada de uma série de aspectos e variáveis de interesse.

Uma limitação de grande parte dos estudos realizados até o presente momento é que, muitas vezes, abordam apenas os cuidadores das crianças e dos adolescentes. Ao se obter apenas um ponto de vista sobre as questões investigadas, perdem-se informações valiosas das crianças e dos jovens que poderiam auxiliar no planejamento de intervenções mais adequadas. Dessa forma, sugere-se investigar suas percepções, necessidades e dificuldades específicas.

\section{Referências}

Abadía-Barrero, C. E., \& LaRusso, M. (2006). The disclosure model versus a developmental illness experience model for children and adolescents living with HIV/Aids in São Paulo, Brazil. Aids Patient Care and STDs, 20, 36-43.

Ayres, J. R. C. M., Paiva, V., França, I., Jr., Gravato, N., Lacerda, R., DellaNegra, M., Marques, H. H. S., Galano, E., Lecussan, P., Segurado, A. A. C., \& Silva, M. H. (2006).Vulnerability, human rights, and comprehensive health care needs of young people living with HIV/Aids. American Journal of Public Health, 96, 1001-1006.

Bachanas, P. J., Kullgren, K. A., Schwartz, K. S., Lanier, B., McDaniel, S., Smith, J., \& Nesheim, S. (2001). Predictors of psychological adjustment in school-age children infected with HIV. Journal of Pediatric Psychology, 26, 343-352. 
Berrien, V. M., Salazar, J. C., Reynolds, E., \& McKay, K. (2004). Adherence to antiretroviral therapy in HIV-infected pediatric patients improve with home-based intensive nursing intervention. Aids Patient Care and STDs, 18, 355-363.

Brackis-Cott, E., Mellins, C. A., Abrams, E., Reval, T., \& Dolezal, C. (2003). Pediatric HIV medication adherence: The views of medical providers from two primary care programs. Journal of Pediatric Health Care, 17, 252-260.

Branco, C. M. (2007). Adesão ao tratamento anti-retroviral por cuidadores de crianças e adolescentes soropositivos de uma unidade de saúde do Estado do Pará. Dissertação de mestrado não-publicada, Universidade Federal do Pará, Belém, PA.

Brasil. Ministério da Saúde. Secretaria de Vigilância em Saúde. Programa Nacional de DST e Aids. (2007). Guia de tratamento clínico da infecção pelo HIV em pediatria (3a ed., Série Manuais, No. 18). Brasília: Ministério da Saúde.

Brown, L. K., \& Lourie, K. J. (2000). Children and adolescents living with HIV and Aids: A review. Journal of Child Psychology and Psychiatry, 41, 81-96.

Byrne, M., Honig, J., Jurgrau, A., Heffernan, S., \& Donahue, M. C. (2002). Achieving adherence with antiretroviral medications for pediatric disease. Aids Read, $12,151-164$.

Duffy, L. (2005). Sufferig, shame and silence: The stigma of HIV/Aids. Journal of the Association of Nurses in Aids Care, 16(1), 13-20.

Ferreira, R., \& Gadelha, G. (2004). Vida que te quero viva: Sistematizando experiências apoiadas pelo POMMAR/ USAID Partners na área de atendimento a crianças que vivem e convivem com o HIV/Aids. Brasília: Expressão Gráfica.

Gerson, A. C., Joyner, M., Fosarelli, P., Butz, A., Wissow, L., Lee, S., Marks, P., \& Hutton, N. (2001). Disclosure of HIV diagnosis to children: When, where, why and how. Journal of Pediatric Health Care, 15, 161-167.

Grecca, K. R. R. (2004). Variáveis identificadas na revelação do diagnóstico de HIV/Aids para crianças e adolescentes. Dissertação de mestrado não-publicada, Pontifícia Universidade Católica de Campinas, Campinas, SP.

Instone, S. L. (2000). Perceptions of children with HIV infection when not told for so long: Implications for diagnosis disclosure. Journal of Pediatric Health Care, 14, 235-243.

Klunklin, P., \& Harrigan, R. C. (2002). Child-rearing practices of primary caregivers of HIV-infected children: An integrative review of the literature. Journal of Pediatric Nursing, 17, 289-296.

Marques, H. H. S., Silva, N. G., Gutierrez, P. L., Lacerda, R., Ayres, J. R. C. M., DellaNegra, M., França I., Jr, Galano, E., Paiva, V., Segurado, A. A. C., \& Silva, M. H. (2006). A revelação do diagnóstico na perspectiva dos adolescentes vivendo com HIV/Aids e seus pais e cuidadores. Cadernos de Saúde Pública, 22, 619-629.
Martin, S. M., Elliott-DeSorbo, D. K., Wolters, P. L., Toledo-Tamula, M. A., Roby, G., Zeichner, S., \& Wood, L.V. (2007). Patient, caregiver and regimen characteristics associated with adherence to highly active antiretroviral therapy among HIV-infected children and adolescents. The Pediatric Infectious Disease Journal, 26, 61-67.

Mellins, C. A., Brackis-Cott, E., Dolezal, C., Richards, A., Nicholas, S. W., \& Abrams, E. J. (2002). Patterns of HIV status disclosure to perinatally HIV-infected children and subsequent mental health outcomes. Clinical Child Psychology and Psychiatry, 7, 101-114.

Naar-King, S., Templin, T., Wright, K., Frey, M., Parsons, J. T., \& Lam, P. (2006). Psychosocial factors and medication adherence in HIV positive youth. Aids Patient Care and STDs, 20, 44-47.

Pequegnat, W. (2002). Research issues with children infected and affected with HIV and their families. Clinical Child Psychology and Psychiatry, 7, 7-15.

Pinto, J. A. (2004, Janeiro). Adolescentes e o tratamento anti-retroviral. Saber viver (Edição especial), 17.

Seidl, E. M. F., Rossi, W. S. R., Viana, K. F., Meneses, A. K. F., \& Meireles, E. (2005). Crianças e adolescentes vivendo com HIV/Aids e suas famílias: Aspectos psicossociais e enfrentamento. Psicologia: Teoria e Pesquisa, 21, 279288.

Swendeman, D., Rotheram-Borus, M. J., Comulada, S., Weiss, R., \& Ramos, M. E. (2006). Predictor's os HIVrelated stigma among young people living with HIV. Health Psychology, 25, 501-509.

Thorne, C., Newell, M. L., Botet, F. A., Bhlin, A. B., Ferrazin, A., Giaquinto, C., Gomez, I. J., Mok, J. Y. Q., Mur, A., \& Peltier, A. (2002). Older children and adolescents surviving with vertically acquired HIV infection. Journal of Acquired Immuno Deficiency Syndrome, 29, 396-401.

United Nations Programme on HIV/Aids (2007). Reducing HIV stigma and discrimination: A critical part of national Aids programmes. Genebra, Suíça: Joint United Nations Programme on HIV/Aids.

Wiener, L. S., \& Battles, H. B. (2006). Untangling the web: A close look at diagnosis disclosure among HIV-infected adolescents. Journal of Adolescent Health, 38, 307-309.

World Health Organization. (2003). Adherence to long term therapies: Evidence for action. Genebra, Suíça: World Health Organization.

Camila Peixoto Pessôa Guerra é psicóloga, Mestre em Psicologia da Saúde pelo Programa de Pós-graduação em Desenvolvimento Humano e Saúde do Instituto de Psicologia da Universidade de Brasília, bolsista CAPES.

Eliane Maria Fleury Seidl é Professora Adjunta do Instituto de Psicologia da Universidade de Brasília.

Recebido: $25 / 04 / 2008$

$1^{a}$ revisão: $20 / 06 / 2008$

$2^{a}$ revisão: 02/03/2009

Aceite final: 02/04/2009 